\title{
Fast cartoon + texture image filters*
}

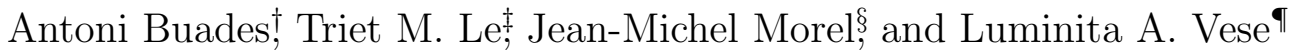

\begin{abstract}
Can images be decomposed into the sum of a geometric part and a textural part? In a theoretical breakthrough, Yves Meyer [28] proposed variational models that force the geometric part into the space of functions with bounded variation, and the textural part into a space of oscillatory distributions. Meyer's models are simple minimization problems extending the famous total variation model. However, their numerical solution has proved challenging. It is the object of a literature rich in variants and numerical attempts. This paper starts with the linear model, which reduces to a low-pass/high-pass filter pair. A simple conversion of the linear filter pair into a non-linear filter pair involving the total variation is introduced. This new-proposed nonlinear filter pair retains both the essential features of Meyer's models and the simplicity and rapidity of the linear model. It depends on only one transparent parameter: the texture scale, measured in pixel mesh. Comparative experiments show a better and faster separation of cartoon from texture. One application is illustrated: edge detection.

Note to the editor in charge and the referees. The algorithm proposed in this paper is tested in the web site

http: //mw. cmla. ens-cachan. fr/megawave/algo/cartoon_texture/

showing many more experiments. An on line demo

http: //mw. cmla. ens-cachan. fr/megawave/demo/cartoon_texture/ permits to test arbitrary images.
\end{abstract}

\section{Introduction to the cartoon + texture problem and prior work}

A grey level or color image will be denoted by $f:(x, y) \in \Omega \rightarrow \mathbb{R}$ (respectively $\mathbb{R}^{3}$ ) where $\Omega$ is an open subset of $\mathbb{R}^{2}$, typically a rectangle or a square. An image $f$ is defined on a continuous domain by interpolating a digital image defined on a finite set of pixels. We are interested in decomposing $f$ into two components $f=u+v$, such that $u$ represents a cartoon or geometric (piecewise-smooth) component of $f$, while $v$

${ }^{*}$ This work has been supported by the National Science Foundation Grants DMS-0714945 and DMS0312222. Research partially financed by the Centre National d'Etudes Spatiales, and the Office of Naval research under grant N00014-97-1-0839.

${ }^{\dagger}$ MAP5, CNRS - Université Paris Descartes, Paris, France (toni.buades@uib.es).

${ }^{\ddagger}$ Mathematics Department, Yale University, New Haven, U.S.A. (triet.le@yale.edu).

${ }^{\S}$ Centre de Mathématiques et de Leurs Applications, École Normale Supérieure de Cachan, France (morel@cmla.ens-cachan.fr).

${ }^{\top}$ Mathematics Department, University of California at Los Angeles, Los Angeles, U.S.A. (lvese@math.ucla.edu). 
represents the oscillatory or textured component of $f$. The oscillatory part $v$ should contain essentially the noise and the texture.

The general variational framework for decomposing $f$ into $u+v$ is given in Meyer's models as an energy minimization problem

$$
\inf _{(u, v) \in X_{1} \times X_{2}}\left\{F_{1}(u)+\lambda F_{2}(v): f=u+v\right\},
$$

where $F_{1}, F_{2} \geq 0$ are functionals and $X_{1}, X_{2}$ are spaces of functions or distributions such that $F_{1}(u)<\infty$ and $F_{2}(v)<\infty$ if and only if $(u, v) \in X_{1} \times X_{2}$. The constant $\lambda>0$ is a tuning parameter. A good model for (1) is given by a choice of $X_{1}$ and $X_{2}$ so that if $u$ is cartoon and if $v$ is texture, then $F_{1}(u)<<F_{2}(u)$ and $F_{1}(v)>>F_{2}(v)$ (such conditions would insure a clear cartoon+texture separation; in other words, if $u$ is only cartoon, without texture, then texture components must be penalized by $F_{1}$, but not by $F_{2}$, and vice-versa).

The long story of this problem can be summarized in a list of proposed choices for both spaces $X_{1}$ and $X_{2}$, and both functionals $F_{1}(u)$ and $F_{2}(v)$. In fact the choice for $F_{1}(u)$ has quickly converged to the total variation of $u$, that excludes strong oscillations but permits sharp edges. The main point under discussion has been what space $X_{2}$ would model the oscillatory part. Since the discussion is complex, we refer to Table 1 and its legend, which present the main models. This table extends the model classification outlined in [11], and adopts the same terminology.

One of the first nonlinear cartoon+texture models is the Mumford and Shah model [30], [31] for image segmentation, where $f \in L^{2}(\Omega)$ is decomposed into $u \in S B V(\Omega)$ ([12], [2], [29], [3]), a piecewise-smooth function with its discontinuity set $J_{u}$ included in a union of curves whose overall length is finite, and $v=f-u \in L^{2}(\Omega)$ represents the noise or the texture. The minimization problem is

$$
\inf _{(u, v) \in S B V(\Omega) \times L^{2}(\Omega)}\left\{\int_{\Omega \backslash J_{u}}|D u|^{2} d x+\mathcal{H}^{1}\left(J_{u}\right)+\lambda\|v\|_{L^{2}(\Omega)}^{2}, f=u+v\right\},
$$

where $\mathcal{H}^{1}$ denotes the 1-dimensional Hausdorff measure (the length if $J_{u}$ is sufficiently smooth), and $\lambda>0$ is a tuning parameter. With the above notations, $X_{1}=S B V(\Omega)$ is the De Giorgi space of special functions with bounded variation. $F_{1}$ is composed of the first two terms in the energy from (2), while the third term is $F_{2}(v)=\int v^{2}$, the quadratic norm. It is difficult to solve this model in practice, because of its non-convex nature coming from $F_{1}(u)$.

An easier decomposition can be obtained by the Rudin, Osher, and Fatemi (ROF) total variation (TV) minimization model [37] for image denoising. Their functional is convex and therefore more amenable to efficient minimization. The variational model is

$$
\inf _{(u, v) \in B V(\Omega) \times L^{2}(\Omega)}\left\{\int_{\Omega}|D u|+\lambda\|v\|_{L^{2}(\Omega)}^{2}, \quad f=u+v\right\},
$$

where

$$
\int_{\Omega}|D u|=\sup \left\{\int_{\Omega} u \operatorname{div} \vec{\phi} d x, \quad \vec{\phi} \in C_{0}^{1}\left(\Omega, \mathbb{R}^{2}\right), \quad\|\vec{\phi}\|_{\infty} \leq 1\right\}
$$

denotes the total variation of $u$ in $\Omega$, also denoted by $T V(u)$ or by $|u|_{B V(\Omega)}$. The component $u$ belongs to the space of functions of bounded variation $B V(\Omega)=\{u \in$ 
$\left.L^{1}(\Omega): \int_{\Omega}|D u|<\infty\right\}$. This space penalizes oscillations (such as noise or texture), but allows for piecewise-smooth functions, made of homogeneous regions with sharp boundaries. Since almost all level lines (or isolines) of a $B V$ function have finite length, the $B V$ space is considered adequate to model images containing shapes. These shapes can actually be extracted by edge detection or by image binarization and morphology $[38])$.

The bibliography on algorithms minimizing the ROF functional and its multi-scale variants $[41,43]$ is rich $[6,44,21,33]$. Convex dual numerical methods have been tested in $[15,32]$. Hybrid models with wavelets are described in $[27,26]$. Models where the $L^{2}$ norm is replaced by the $L^{1}$ norm are now classical [16].

In [17] strong mathematical geometric arguments are put forward in favor of the $T V$ - $L^{1}$ model: explicit solutions can be computed for simple geometric objects. These examples demonstrate that, based on the perimeter/area ratio, shapes are unambiguously put either in the TV part or in the $L^{1}$ part. This study connects the $T V-L^{1}$ model with the classical morphological granulometry [38]. Accurate regularity results for the level set boundaries of minimizers of the $T V-L^{1}$ model are also given, in any dimension, in [1]. Probably the most popular $T V$ minimization algorithm is Chambolle's projection algorithm [14]. Recent years have, however, shown a trend to abandon the $B V$ norm and replace it by a so-called "non-local" norm [34] inspired from [13].

Yet, as pointed out in [28], $T V-L^{2}$ or $T V-L^{1}$ do not characterize the oscillatory components. Indeed, these components do not have small norms in $L^{p}(\Omega), p \geq 1$, [4]. To overcome this drawback, Y. Meyer [28] proposed in his seminal book weaker norms to replace $\|\cdot\|_{L^{2}}^{2}$ in the ROF model, that would better model oscillatory components with zero mean. The Meyer model is

$$
\inf _{(u, v) \in(B V(\Omega) \times G, F \text { or } E), f=u+v}\left\{\int_{\Omega}|D u|+\lambda\|v\|_{*}\right\},
$$

where $\|\cdot\|_{*}$ is the norm in one of the following spaces, denoted by $G, F$ or $E$ (defined here for $\Omega=\mathbb{R}^{2}$ ).

Definition $1 A$ distribution $v$ belongs to $G$ if and only if $v=\operatorname{div}(\vec{g})$ for some $\vec{g} \in$ $\left(L^{\infty}\right)^{2}$ in the distributional sense. The endowed norm is

$$
\|v\|_{*}=\|v\|_{G}=\inf _{\vec{g} \in L^{\infty}, v=\operatorname{div} \vec{g}}\|\vec{g}\|_{L^{\infty}} .
$$

The space $F$ is defined as $G$, but the condition $\vec{g} \in\left(L^{\infty}\right)^{2}$ is substituted by the weaker condition $\vec{g} \in B M O^{2}$ (thus if $\vec{g}=\left(g_{1}, g_{2}\right)$, then $g_{i}$ are functions with bounded mean oscillation). Finally, the space $E$ is the Besov space $E=\dot{B}_{\infty, \infty}^{-1}$, dual to the space $\dot{B}_{1,1}^{1}$.

The introduction of the spaces $G, F$ and $E$ is motivated by the fact that highly oscillatory signals or images have small norms in $G, F$ or $E$. For instance, $\|\cos n x\|_{G}=$ $\frac{1}{n}$. The presence of a non- $B V$ part in images is corroborated by the experimentalnumerical study [20]. However, the three norms proposed by Meyer are not expressed as integrals and are therefore difficult to compute. It is also difficult to set up the right value of $\lambda$ for real images. This problem is addressed in [42] and [10]. The numerical experiments have shown promising results and justified further inquiries. 


\begin{tabular}{|c|c|c|}
\hline Minimized energy or filters & Name & Reference \\
\hline $\int|D u|^{2}+\mathcal{H}^{1}\left(J_{u}\right)+\int|v|^{2}$ & $S B V-L^{2}(\mathrm{MS})$ & {$[30]$} \\
$\int|D u|+\int|v|^{2}$ & $T V-L^{2}(\mathrm{ROF})$ & {$[37]$} \\
$\int|D u|+\int|v|$ & $T V-L^{1}$ & {$[16]$} \\
\hline $\int|D u|+\inf _{\vec{g} \in L^{\infty}, v=\operatorname{div} \vec{g}\|\vec{g}\|_{L^{\infty}}}$ & $T V-\operatorname{div}\left(L^{\infty}\right)$ & {$[28]$} \\
$\int|D u|+\|v\|_{H^{-1}}$ & $\mathrm{TV}-H^{-1}$ & {$[36]$} \\
$\int|D u|+\inf _{\vec{g} \in B M O, v=\operatorname{div} \vec{g}\|\vec{g}\|_{L^{\infty}}}$ & $\mathrm{TV}-\operatorname{div}(\mathrm{BMO})$ & {$[28,23,18]$} \\
$\int|D u|+\|v\|_{\dot{B}_{\infty}^{-1}, \infty}$ & $\mathrm{TV}-$ Besov & {$[28,19,9]$} \\
\hline $\int|D u|+\int|K * v|^{2}$ & TV-Hilbert & {$[11]$} \\
$\int|D u|^{2}+\|v\|_{H^{-1}}^{2}$ & $H^{1}-H^{-1}$ & Here and [39] \\
\hline$u=w L_{\sigma} * f+(1-w) f$ & nonlinear filter pair & Here \\
\hline
\end{tabular}

Table 1: Table of all $f=u+v=$ cartoon + texture models in approximate chronological order. These models are divided in five groups. The first group contains the classic $B V$ or $S B V+$ noise models. The second group starting with Meyer's model introduces a key new feature: The norm of the oscillatory part $v$ decreases when $v$ oscillates more. This is obtained by putting a norm on $v$ that is actually a norm on a primitive of $v$. The TV- $H^{-1}$, TV-div(BMO) and TV-Besov models follow the same pattern. The third group simplifies the panorama by pointing out that the norm of a primitive of $v$ is much easier to compute by convolution with a filter $K$ (in fact the TV- $H^{-1}$ model also belongs to that group). But here, the main fact is that the second model in the third group, $H^{1}-H^{-1}$, boils down to the decomposition into a classic low-pass and high-pass decomposition. As will be shown in Sect. 4 such linear decompositions do give competitive results. The last row is the proposed nonlinear filter, which takes the best of each worlds by using $B V$, but relying mainly on a previous pair of linear high-pass and low-pass filters.

There has been an extensive line of papers (starting with [46]) modifying and interpreting Meyer's models, and proposing minimization schemes: [7, 40, 9, 47, 24, 45, 22]. An extensive mathematical analysis of Meyer's model in a bounded domain is performed in [5]. For many formal properties of the $G$-norm the reader can refer to [35]. In [36] the $G$-norm is replaced by the $H^{-1}$ norm. This approach using Sobolev spaces with negative exponents was extended in [25] and [18]. The $F=\operatorname{div}(B M O)$ variant was numerically studied in [23] and [18]. There have also been extensions intending to decompose $u$ into three components, namely $B V$, texture, and a residual (e.g., noise). In the model [46] (where the space $G=\dot{W}^{-1, \infty}$ is approximated by $G_{p}=\dot{W}^{-1, p}$ for large $p$ ), this is done by solving

$$
\inf \int_{\Omega}|D u|+\lambda\|f-u-v\|_{L^{2}(\Omega)}^{2}+\alpha\|v\|_{G_{p}} .
$$

In [46] the norm $\|v\|_{G}$ of $v=\operatorname{div} \vec{g}$ is approximated by $\left\|\sqrt{g_{1}^{2}+g_{2}^{2}}\right\|_{p}, p \geq 1$ which is of course far from the real problem with $p=\infty$. Aujol et al. [8] addressed the original Meyer problem and proposed an alternate method to minimize

$$
\inf \int_{\Omega}|D u|+\lambda|| f-u-v \|_{L^{2}(\Omega)}^{2}
$$

subject to the constraint $\|v\|_{G} \leq \mu$. 
The 2006 paper [11] presents a sort of review where the above mentioned variants and others are summarized. Following this paper's terminology, the funding models that inspired this line of research are $T V-L^{2}$ (ROF) and the original Meyer models $T V-$ $\operatorname{div}\left(L^{\infty}\right), T V-\operatorname{div}(B M O)$ (numerically tried in [23], [18]), and $T V$-Besov (numerically tried in [19], [9]). A simpler variant is $T V-H^{-1}$, since also the $H^{-1}$ norm is small on oscillatory signals. The hierarchy of the spaces used for the oscillatory part is complex: $\operatorname{div}\left(L^{\infty}\right)$ and $\operatorname{div}(B M O)$ are distributional first derivatives of vector fields in $L^{\infty}$ and $B M O$ respectively. The Besov model takes the oscillatory part $v$ into $\dot{B}_{\infty, \infty}^{-1}:=\Delta\left(\dot{B}_{\infty, \infty}^{1}\right)$ which is a space of second derivatives of functions satisfying a Zygmund regularity condition. Since this condition is close to assuming a Lipschitz bound on the functions, it is fair to say that the Besov model defines distributions that are second derivatives of functions that have (almost) bounded gradients.

In conclusion (as also pointed out by $Y$. Meyer [28]), the four spaces $G=\operatorname{div}\left(L^{\infty}\right)$, $H^{-1}=\Delta\left(H^{1}\right), F=\operatorname{div}(B M O)$ and $\Delta\left(\dot{B}_{\infty, \infty}^{1}\right)$ (Besov) can be considered as variants of each other, since they all appear as first derivatives of (bounded-like) functions. Experimental evidence does not favor one of them.

Generalizing $T V-H^{-1}$, a generic $T V$-Hilbert model [11] can be defined using a smoothing kernel $K$. The associated Meyer energy is

$$
\inf _{u \in B V}\left\{\mathcal{J}(u)=\int|D u|+\lambda\|K *(f-u)\|_{L^{2}}^{2}\right\} .
$$

This model has also been proposed in [19]. The $L^{2}$ norm of $K *(f-u)$ can be substituted by an $L^{p}$ norm, $p \geq 1$. One obtains slightly better results with $p=1$ [18]. Our numerical trials yield no significant difference between $T V$-Hilbert and the other mentioned $T V-X$ models. Because of its simplicity, we shall retain this version (5) in the experiments after fixing adequately the kernel $K$. This is precisely the object of the next section. The main goal of the manuscript is to propose here a simpler and faster model than the variational model (5), while better separating cartoon from texture.

We wish to recall here the function spaces notations used in the next sections. $H^{0}=L^{2}$ denotes the space of square-integrable functions. The Sobolev space $H^{1}$ is defined by $H^{1}=\left\{u \in L^{2}, D u \in L^{2} \times L^{2}\right\}$, or in the Fourier domain by $H^{1}=\{u$ : $\left.\int\left[1+(2 \pi|\xi|)^{2}\right]|\hat{u}(\xi)|^{2} d \xi<\infty\right\}$. We will also make use of the space $H^{-1}$ (dual to the homogeneous version of $H^{1}$ ), defined in the Fourier domain by the set of functions and distributions $H^{-1}=\left\{u: \int\left[1+(2 \pi|\xi|)^{2}\right]^{-1}|\hat{u}(\xi)|^{2} d \xi<\infty\right\}$ (the corresponding homogeneous versions, used in the next sections, are obtained by dropping the constant 1).

The rest of the paper is organized as follows: in Section 2 we formulate the linear cartoon + texture $H^{1}-H^{-1}$ model inspired from Y. Meyer [28], which can be easily and rapidly solved in the Fourier domain in one step. Since this model introduces blurring in the cartoon component $u$, we propose in Section 3 a novel nonlinear cartoon + texture model that retains the simplicity and efficiency of the linear one, while the cartoon component $u$ is piecewise-smooth and with sharp edges. Section 4 illustrates numerical comparisons between the linear model, the nonlinear minimization model (5) and the proposed fast nonlinear model; an application to edge detection is also shown, together with a discussion on the local texture scale. 


\section{Linear version of Meyer's model}

In view of the multiplicity and complexity of nonlinear models, it seems reasonable to first fix as a reference the best linear model. Separation of scales in images is classically obtained by applying a complementary pair of low-pass and high-pass filters to the data $f$, namely $u=L P F(f)$, and then $v=f-u=H P F(f)$. The $T V-H^{1}$ model is easily linearized by replacing the total variation $\int|D u|$ by the Dirichlet integral $\int|D u|^{2}$. Then the most natural variational linear model associated with Meyer's ideas is $H^{1}-H^{-1}$. Indeed, $H^{-1}$ is dual to $H^{1}$, in the same way as $G$ is dual to $B V$. The low pass filter $f \rightarrow u$ is obtained by the minimization

$$
\min _{u}\left\{\sigma^{4} \int|D u|^{2}+\|f-u\|_{H^{-1}}^{2}\right\} .
$$

The meaning of $\sigma^{4}$ will be shortly explained. This model can be compared with the classical Tikhonov quadratic $H^{1}-L^{2}$ minimization

$$
\min _{u}\left\{\sigma^{2} \int|D u|^{2}+\int(f-u)^{2}\right\}
$$

which is equivalent in the Fourier domain to the low-pass filter $\hat{u}=\frac{1}{1+(2 \pi \sigma|\xi|)^{2}} \hat{f}$. This Wiener filter is known to remove high-frequency components due to the edges of $f$, and not only those due to oscillations (See Fig. 1).

Using the Fourier transform in (6), the $H^{1}$ semi-norm of $u$ is $\int|D u|^{2}=\int(2 \pi|\xi|)^{2}|\hat{u}(\xi)|^{2}$ and the $H^{-1}$ semi-norm of $v$ is $\int \frac{|\hat{v}(\xi)|^{2}}{(2 \pi|\xi|)^{2}}$. This implies in particular that $u-f=v$ has zero mean, since feasible solutions satisfy $\hat{v}(0)=0$. Minimizing this quadratic functional (6) in $u$ yields in Fourier the unique solution $\hat{u}=\hat{L}_{\sigma} \hat{f}$, where

$$
\hat{L}_{\sigma}(\xi):=\frac{1}{1+(2 \pi \sigma|\xi|)^{4}} .
$$

The meaning of the parameter $\sigma$ is now easily explained: if the frequency $\xi$ is significantly smaller than $\frac{1}{2 \pi \sigma}$, then the $\xi$ frequency is kept in $u$, while if $\xi$ is significantly larger than $\frac{1}{2 \pi \sigma}$, then the frequency $\xi$ is considered a textural frequency and attributed to $v$. Thus, the solution $(u, v)=\left(L_{\sigma} * f,\left(I d-L_{\sigma}\right) * f\right)$ is nothing but a pair of complementary low pass and high pass filters. Note that as $\sigma \rightarrow 0, L_{\sigma} \rightarrow I d$. We will also consider the filter $K_{\sigma}$, where $\hat{K}_{\sigma}(\xi)=e^{-(2 \pi \sigma|\xi|)^{4}}$, which behaves still more like the characteristic function of the ball centered at zero with radius $\frac{1}{2 \pi \sigma}$.

It is worth mentioning that related linear and nonlinear three-term decompositions $f=u+v+w$ based on the $H^{1}-H^{-1}$ duality were introduced in [39]: the linear case is the $\left(H^{1}, H^{0}, H^{-1}\right)$ decomposition, while the nonlinear decomposition uses piecewise $\left(H^{1}, H^{0}, H^{-1}\right.$ ) (where piecewise $H^{1}$ is the $S B V$ space for the cartoon, combined with piecewise $H^{-1}$ for the texture).

\section{Proposed fast cartoon+texture non-linear filters}

The observed efficiency of the linear pair $\left(L_{\sigma}, I d-L_{\sigma}\right)$ (see Figure 3 ) leads to consider nonlinear versions that would retain its main feature, namely the excellent extraction 


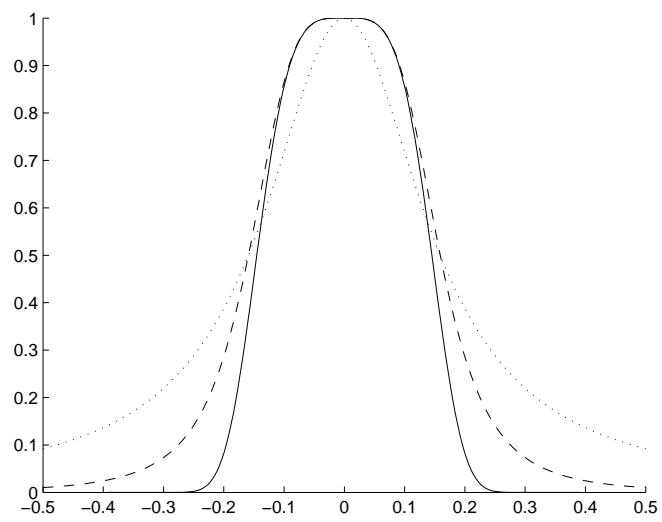

Figure 1: Fourier transform of the low pass filter obtained with the $H^{1}-L^{2}$ functional (7) (dotted line), the filter $L_{\sigma}(8)$ associated with the $H^{1}-H^{-1}$ model (6) (dashed line) and the filter $K_{\sigma}$ (solid line) for $\sigma=1$. Among the three filters, $K_{\sigma}$ behaves more like the sharpest possible low-pass filter, namely the characteristic function of $[-2 \pi \sigma, 2 \pi \sigma]$.

of the texture by a high pass filter $I d-L_{\sigma}$. On the other hand, the non-oscillatory parts of the initial image $f$ should be kept unaltered even if they have sharp edges. This is of course impossible with a linear filter. Thus, a local indicator must be built to decide at each point $x$ whether it belongs to a textural region or to a cartoon region. The main characteristics of a cartoon region is that its total variation does not decrease by low pass filtering. The main characteristics of a textured region is its high total variation due to its oscillations. This total variation decreases very fast under low pass filtering. Formalizing these remarks leads to define the local total variation (LTV) at $x$,

$$
L T V_{\sigma}(f)(x):=L_{\sigma} *|D f|(x)
$$

(note that $L_{\sigma}$ can be substituted by $K_{\sigma}$ ). The relative reduction rate of $L T V$ is defined by a function $x \mapsto \lambda_{\sigma}(x)$, given by

$$
\lambda_{\sigma}(x):=\frac{L T V_{\sigma}(f)(x)-L T V_{\sigma}\left(L_{\sigma} * f\right)(x)}{L T V_{\sigma}(f)(x)}
$$

which gives us the local oscillatory behavior of the function $f$. If $\lambda_{\sigma}$ is close to 0 , we have

$$
\frac{L T V_{\sigma}(f)-L T V_{\sigma}\left(L_{\sigma} * f\right)}{L T V_{\sigma}(f)} \leq \lambda_{\sigma} \Leftrightarrow L T V_{\sigma}\left(L_{\sigma} * f\right) \geq\left(1-\lambda_{\sigma}\right) L T V_{\sigma}(f),
$$

which means that there is little relative reduction of the local total variation by the low pass filter. If instead $\lambda_{\sigma}$ is close to 1 , the reduction is important, which means that the considered point belongs to a textured region. Thus, a fast nonlinear low pass and high pass filter pair can be computed by weighted averages of $f$ and $L_{\sigma} * f$ depending on the relative reduction of $L T V$. We can set

$$
u(x)=w\left(\lambda_{\sigma}(x)\right)\left(L_{\sigma} * f\right)(x)+\left(1-w\left(\lambda_{\sigma}(x)\right)\right) f(x), \quad v(x)=f(x)-u(x)
$$


where $w(x):[0,1] \rightarrow[0,1]$ is an increasing function that is constant and equal to zero near zero and constant and equal to 1 near 1 . In all experiments the soft threshold function $w$ is defined by

$$
w(x)=\left\{\begin{array}{lc}
0 & x \leq a_{1} \\
\left(x-a_{1}\right) /\left(a_{2}-a_{1}\right) & a_{1} \leq x \leq a_{2} \\
1 & x \geq a_{2}
\end{array}\right.
$$

where the parameters $a_{1}$ and $a_{2}$ have been respectively fixed to 0.25 and 0.5 . If $\lambda_{\sigma}(x)$ is small, the function $f$ is non-oscillatory around $x$ and therefore the function is $B V$ (or cartoon) around $x$. Thus $u(x)=f(x)$ is the right choice. If instead $\lambda_{\sigma}(x)$ is large, the function $f$ is locally oscillatory around $x$ and locally replaced by $\left(L_{\sigma} * f\right)(x)$. The choice of $\lambda_{\sigma}=\frac{1}{2}$ as underlying hard threshold is conservative: it permits to keep all step edges on the cartoon side, but puts all fine structures on the texture side, as soon as they oscillate more than once. Of course changes in the parameters $a_{1}$ or $a_{2}$ would slightly modify the separation results.

Since it is desirable to have a one-parameter method, it seems advisable to fix the threshold function $w$ once and for all, as has been done in all experiments. In that way the method keeps the scale $\sigma$ of the texture as the only method parameter. That this last parameter cannot be avoided is obvious: textural details become shapes when their sizes grow, and therefore should be moved from the texture to the $B V$ side. This is apparent in the experiments of Figs. 6 and 7.

\section{Comparing results}

In this section the results of three main representative models will be compared. First, the simplest linear Meyer model, namely the linear $H^{1}-H^{-1}$ model, second the standard $T V-H i l b e r t$ model (5), and finally the fast nonlinear filter defined in Section 3. Implementing the $T V-$ Hilbert model amounts to minimize the energy

$$
\inf _{u \in B V(\Omega)} \int_{\Omega}|D u|+\frac{\lambda}{2} \int_{\Omega}\left|L_{\sigma} *(f-u)(x)\right|^{2} d x
$$

where the smoothing kernel $L_{\sigma}$ will be the same as for the linear and the nonlinear filter pairs, to permit fair comparisons. By gradient descent, $u$ must formally solve

$$
\frac{\partial u}{\partial t}=\operatorname{div}\left(\frac{\nabla u}{|\nabla u|}\right)+\lambda L_{\sigma} * L_{\sigma} *(f-u)
$$

This numerical method is actually slower than the smart methods for minimizing the total variation mentioned in the introduction, but gives essentially the same results.

Figure 3 compares cartoon and texture components for the linear filter, the $T V$ Hilbert formulation, and the proposed non-linear filter pair. Clearly the edges are better preserved in the cartoon part with the proposed non-linear filter, and much less apparent in the texture part. The $H^{1}-H^{-1}$ Meyer linear filter pair gives strikingly good results, but blurs slightly out edges in the cartoon part, as expected. As a consequence, ghosts of the edges appear in the textural part. A careful comparison of $H^{1}-H^{-1}$ with $T V$-Hilbert confirms the slight improvement of the nonlinear variational model on the 

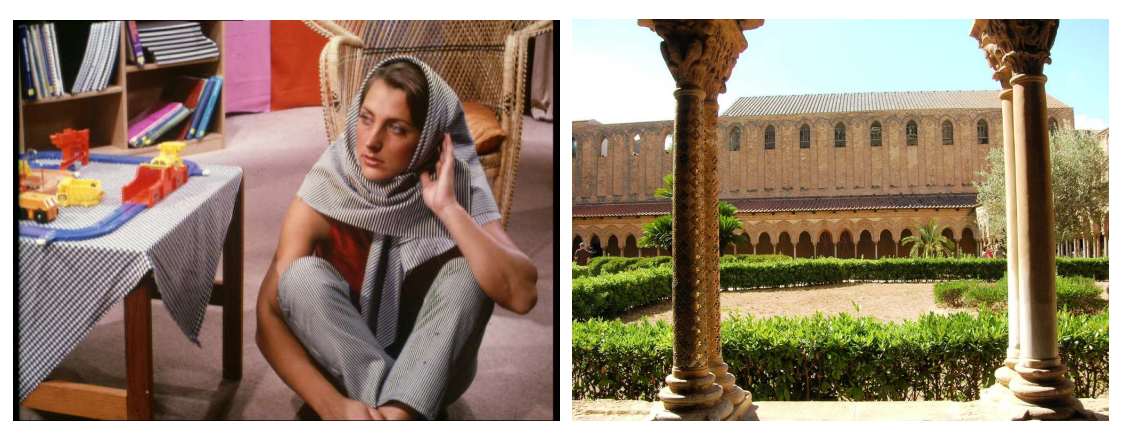

Figure 2: Test images Barbara and patio. We will also use the gray level version of Barbara image.

linear one. Figure 4 displays the plots of $\lambda_{\sigma}(x)$ for several pixels in the Barbara image and different $\sigma$. This figure illustrates how $\lambda_{\sigma}(x)$ increases with $\sigma$ for high frequency textural patterns and gets quickly close to one. On contours and flat zones $\lambda_{\sigma}(x)$ increases very slowly tending to values much lower than 0.5 , thus explaining the chosen values of $a_{1}$ and $a_{2}$ in equation (10).

Figure 5 illustrates the efficiency of the separation of texture from the $B V$ part by applying a Canny filter to the cartoon part $u$ (right) obtained by the proposed nonlinear filter. The edges between textural regions are indeed detected on the cartoon part. If applied directly on the original image (left), these edges are mixed up with numerous texture edges.

A serious advantage of the proposed nonlinear filter is that the Lagrange parameter $\lambda$ in the original Meyer model is now interpreted as a scale $\sigma$. Thus, it is easy to fix $\sigma$ in the low pass filter to put (or not) this texture in the textural part: it is enough to evaluate the wave-length (in pixels) of the texture and to fix $\sigma$ accordingly. In Fig. 6 , the transparent choice of $\sigma$ is shown on the classical textured image Barbara. The micro-textures are put in the oscillatory part for $\sigma=4$, and the larger textures for $\sigma=6$. Eventually, for $\sigma=8$, the oscillations of the books and chair go into the texture part. The function $\lambda_{\sigma}(x)$ used for these decompositions is displayed in Fig. 8.

The sharper kernel $K_{\sigma}$ instead of $L_{\sigma}$ was also tested in the nonlinear filter, as shown in Fig. 7. $K_{\sigma}$ behaving more like a characteristic function, the oscillations on the scarf, the chair and the books are slightly better separated than in the results from Figure 6 using $L_{\sigma}$. But this comparison also shows that the choice of the low-pass filter is not crucial. A final decomposition experiment is displayed in Figure 9. This figure corroborates the efficiency of the separation of texture from the $B V$ part. Notice how the contours of columns and arcades remain sharp in the $B V$ parts. However, the thin columns seen at a distance pass into texture for $\sigma=4$.

\section{References}

[1] W.K. Allard. Total Variation Regularization for Image Denoising, I. Geometric Theory. SIAM Journal on Mathematical Analysis, 39(4):1150-1190, 2008. 

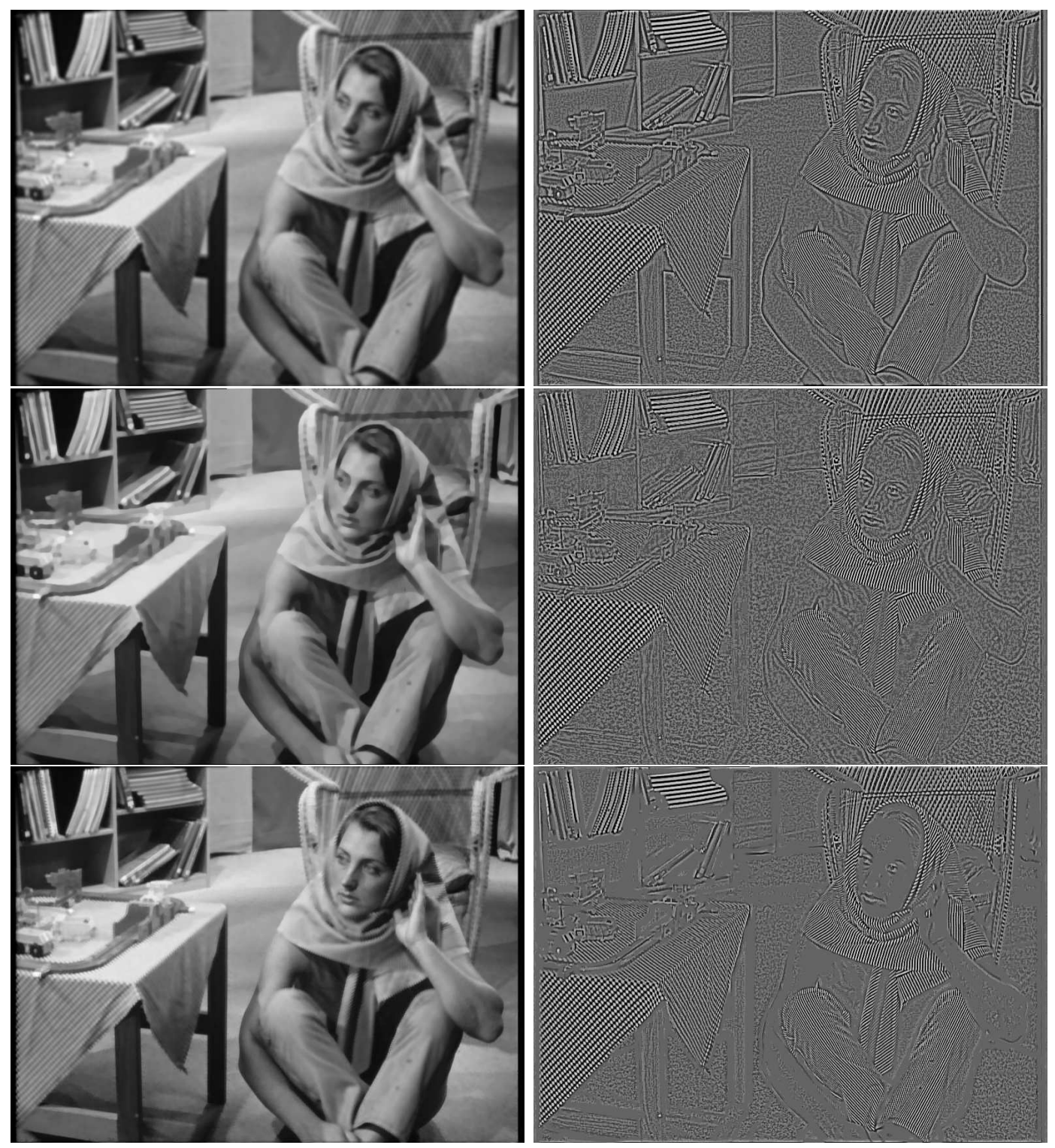

Figure 3: Barbara in gray with a fixed $\sigma=1.5$. Compares cartoon and texture for the linear $H^{1}-H^{-1}$ linear filter, the $T V$-Hilbert formulation, and the proposed non-linear filter pair using $L_{\sigma}$. Observations: 1 ) the linear filter gives quite a good decomposition, but of course makes the cartoon slightly blurry. Remnants of edges appear in the texture image. This is still true for the TV-Hilbert model. The proposed filter eliminates all traces of edges in the texture part, and gives a sharp cartoon; 2) the proposed filter is not obtained by a variational minimization and retains, for instance, a higher energy than the TV-Hilbert solution in the middle row. 

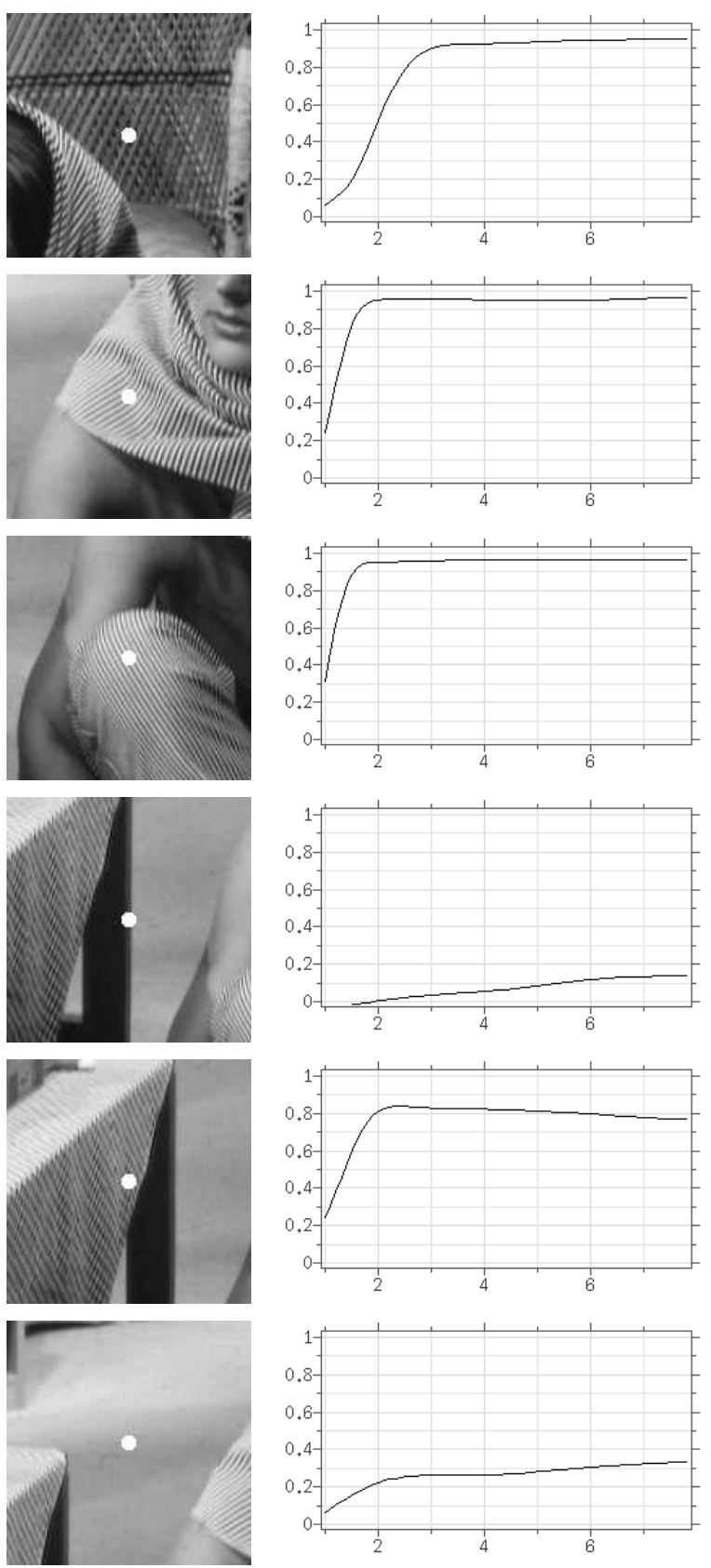

Figure 4: Display of plots $\lambda_{\sigma}(x)$ for several pixels in the Barbara image and different $\sigma$. The function $\lambda_{\sigma}(x)$ increases with $\sigma$ for high frequency textural patterns and gets quickly close to one. On contours and flat zones $\lambda_{\sigma}(x)$ increases very slowly tending to values much lower than 0.5 , thus explaining the chosen values of $a_{1}$ and $a_{2}$ in equation (10). 

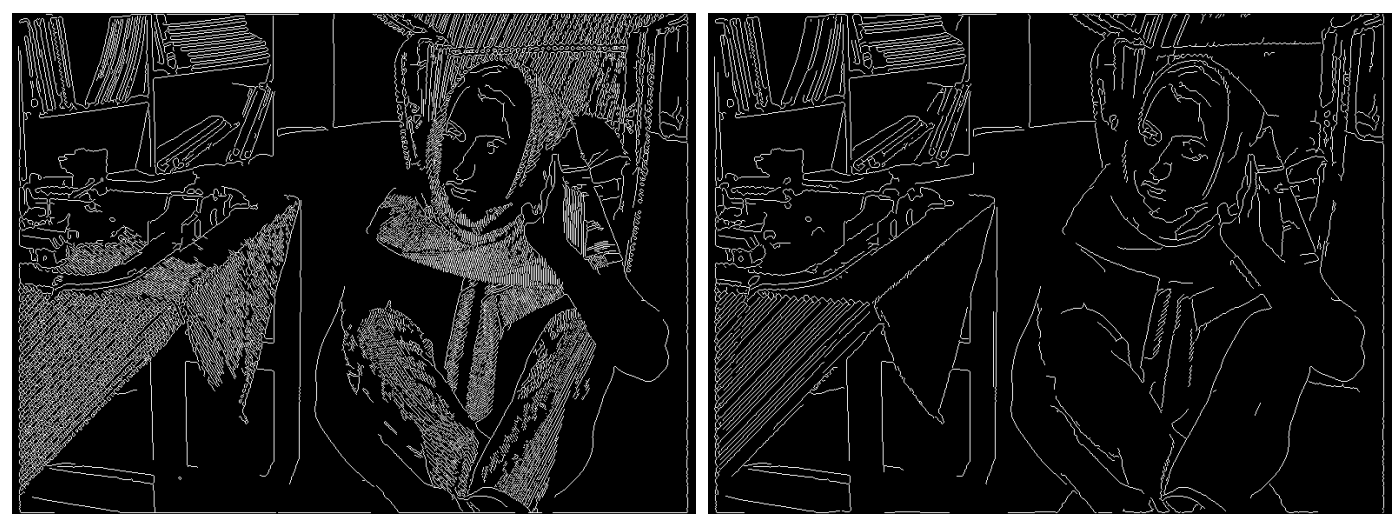

Figure 5: Canny's edge detector applied on the original Barbara image, and Canny's edge detector applied on the cartoon image obtained by the proposed algorithm in Figure 3. Edges caused by the textures are eliminated.

[2] L. Ambrosio. Variational problems in SBV and image segmentation. Acta Applicandae Mathematicae, 17(1):1-40, 1989.

[3] L. Ambrosio, N. Fusco, and D. Pallara. Functions of bounded variation and free discontinuity problems. Oxford University Press, 2000.

[4] F. Andreu-Vaillo, V. Caselles, and J.M. Mazón. Parabolic Quasilinear Equations Minimizing Linear Growth Functionals. Birkhauser, 2004.

[5] G. Aubert and J.F. Aujol. Modeling very oscillating signals. Application to image processing. Applied Mathematics and Optimization, 51(2):163-182, 2005.

[6] G. Aubert and L. Vese. A variational method in image recovery. SIAM J. on Num. Anal., 34:1948-1979, 1997.

[7] J.F. Aujol, G. Aubert, L. Blanc-Féraud, and A. Chambolle. Image decomposition. Application to SAR images. Lecture Notes in Computer Science, 2695:297-312, 2003.

[8] J.F. Aujol, G. Aubert, L. Blanc-Féraud, and A. Chambolle. Image Decomposition into a Bounded Variation Component and an Oscillating Component. Journal of Mathematical Imaging and Vision, 22(1):71-88, 2005.

[9] J.F. Aujol and A. Chambolle. Dual norms and image decomposition models. International Journal of Computer Vision, 63(1):85-104, 2005.

[10] J.F. Aujol and G. Gilboa. Implementation and parameter selection for BV-Hilbert space regularization. UCLA C.A.M. Report, 04-66, 2004.

[11] J.F. Aujol, G. Gilboa, T. Chan, and S. Osher. Structure-texture image decomposition - modeling, algorithms and parameter selection. International Journal of Computer Vision, 67(1):111-136, 2006 (UCLA CAM Report 05-10, 2005). 


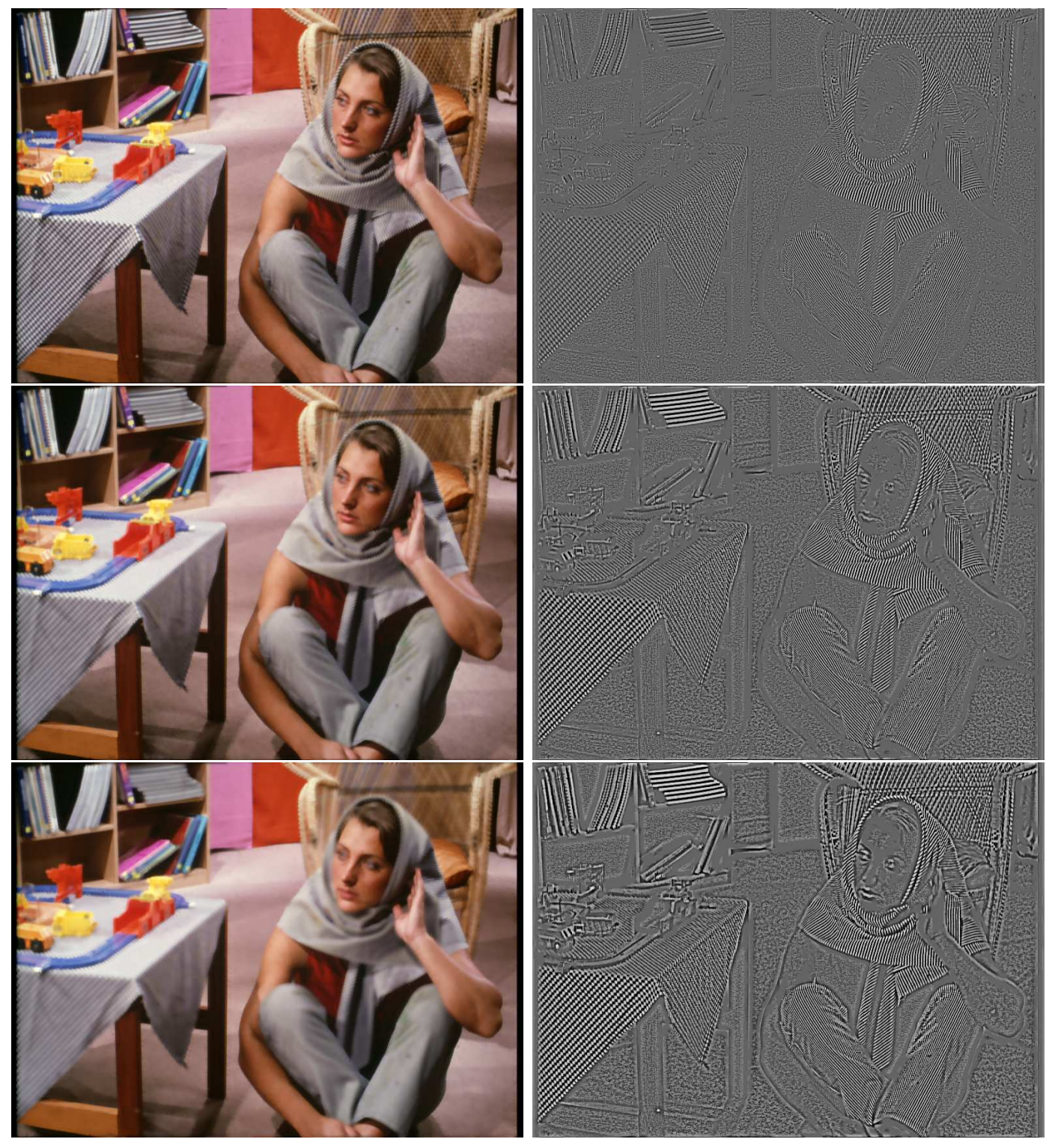

Figure 6: The decomposition using the proposed filter with $L_{\sigma}$ where $\sigma=4,6,8$. 

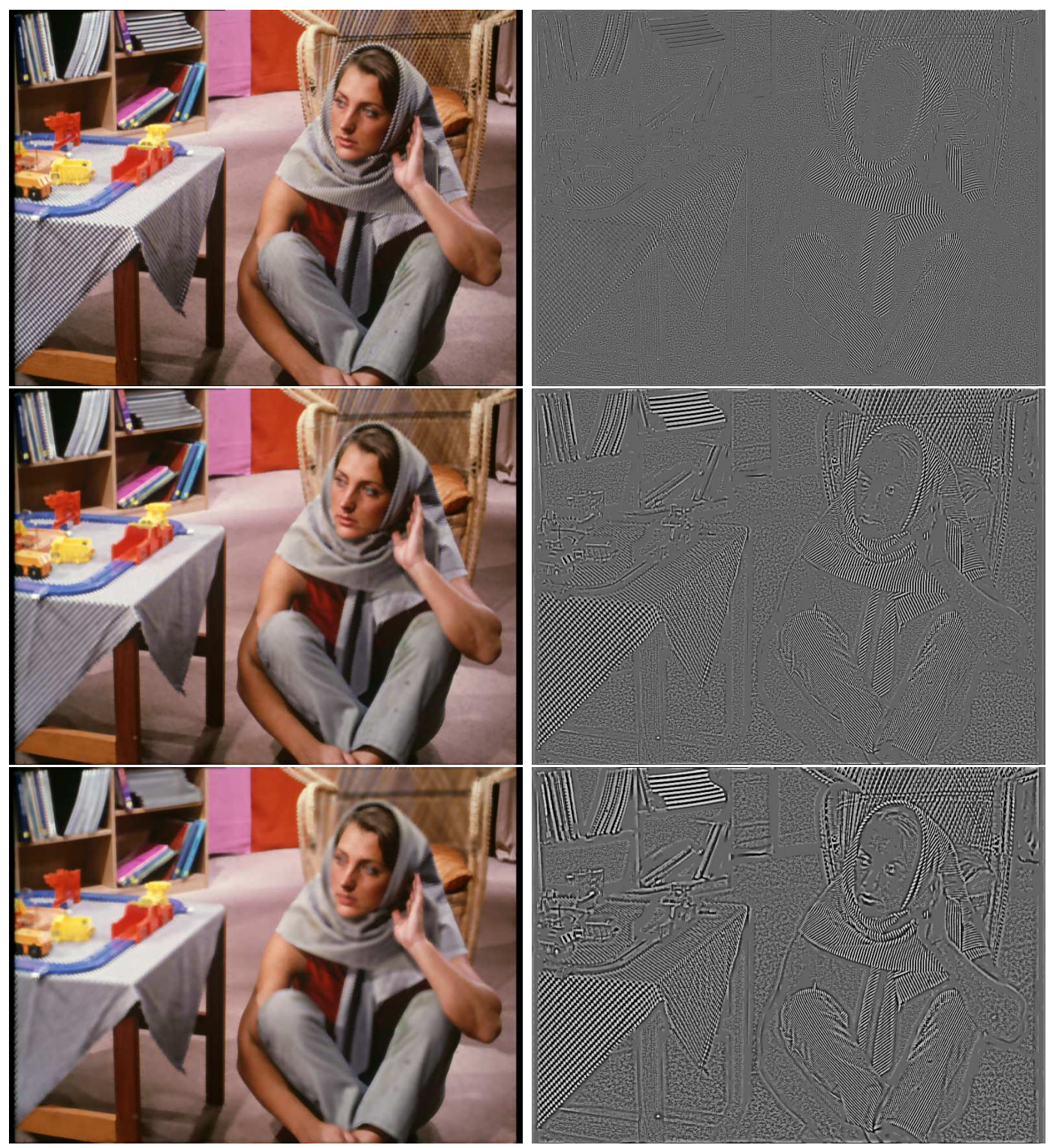

Figure 7: The decomposition using the proposed filter with $K_{\sigma}$ where $\sigma=4,6,8$. It is slightly sharper than with $L_{\sigma}$ : compare with fig. 6 . 


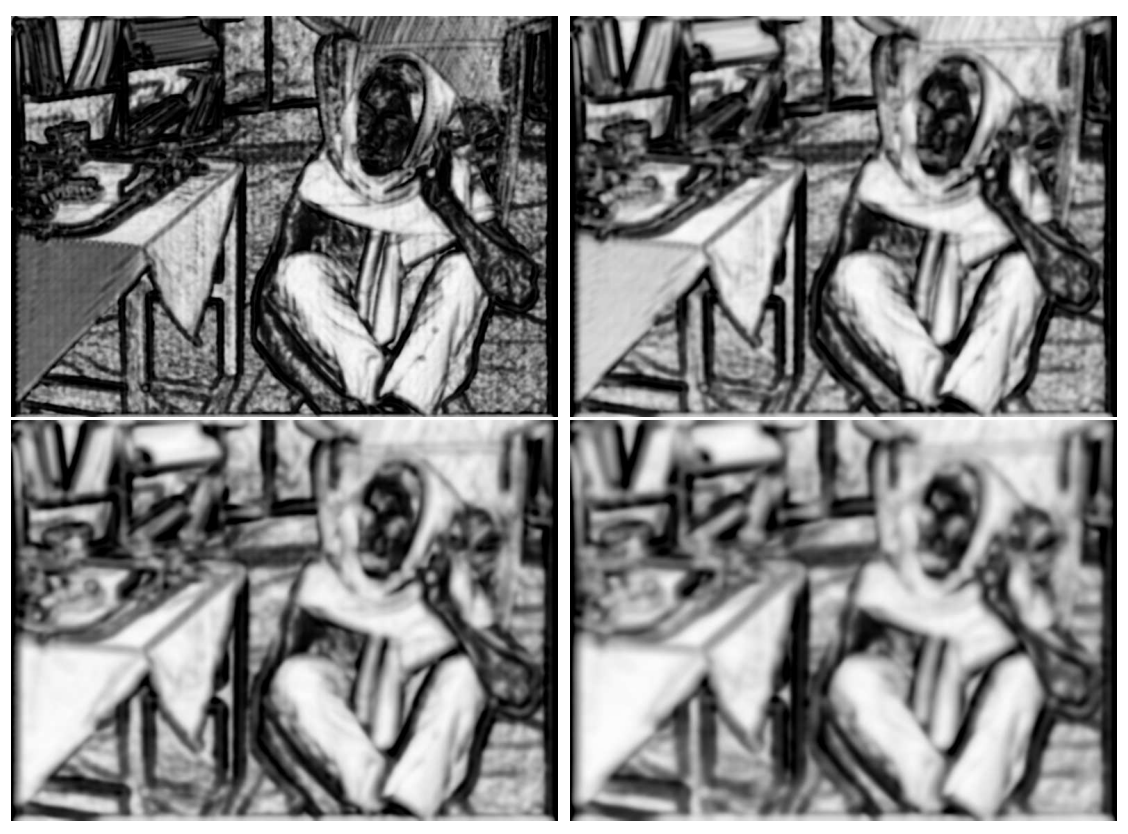

Figure 8: Display of $\lambda_{\sigma}(x)$ used in the decompositions of Fig. 6. Clearly $\lambda$ is large on oscillatory textures and small on edges. See also Fig. 4 for the detailed shape of this function of $\sigma$ at several characteristic points.

[12] A. Blake and A. Zisserman. Visual Reconstruction. The MIT Press Cambridge, Massachusetts, 1987.

[13] A. Buades, B. Coll, and J. M. Morel. A review of image denoising algorithms, with a new one. Multiscale Modeling Simulation, 4(2):490-530, 2005.

[14] A. Chambolle. An algorithm for total variation minimization and applications. Journal of Mathematical Imaging and Vision, 20(1):89-97, 2004.

[15] A. Chambolle and P.L. Lions. Image recovery via total variation minimization and related problems. Numerische Mathematik, 76(2):167-188, 1997.

[16] T.F. Chan and S. Esedoglu. Aspects of total variation regularized $L^{1}$ function approximation. SIAM J. on Appl. Maths., 65(5):1817-1837, 2005.

[17] V. Duval, J.F. Aujol, and Y. Gousseau. The TVL1 model: a geometric point of view. 2009 .

[18] J.B. Garnett, P.W. Jones, T.M. Le, and L.A. Vese. Modeling oscillatory components with the homogeneous spaces $B M O^{-\alpha}$ and $W^{-\alpha, p}$. UCLA CAM Report 07-21, to appear in PAMQ, 2007.

[19] J.B. Garnett, T.M. Le, Y. Meyer, and L.A. Vese. Image decompositions using bounded variation and generalized homogeneous Besov spaces. Applied and Computational Harmonic Analysis, 23(1):25-56, 2007 (UCLA CAM Report 05-57, 2005). 


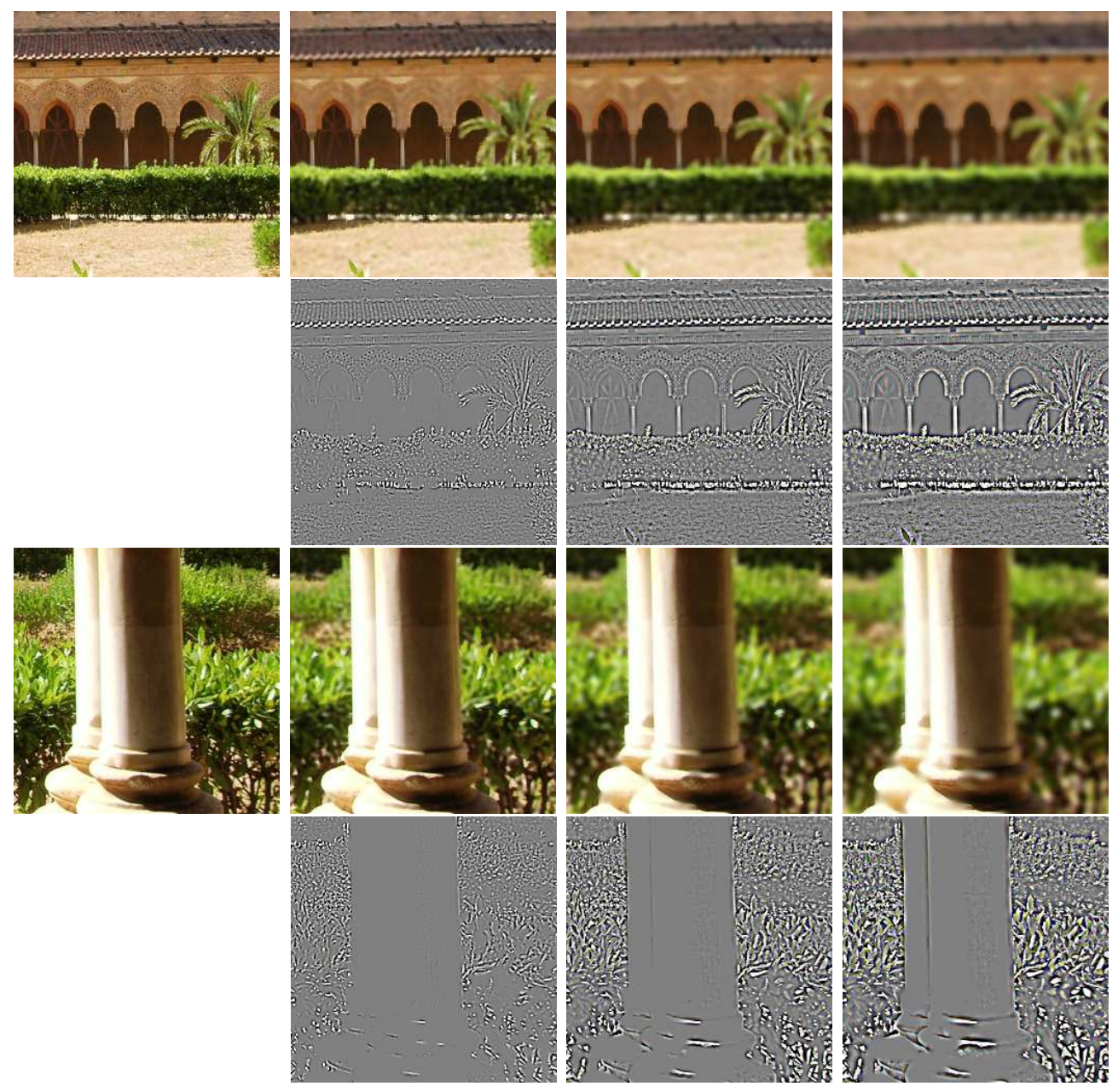

Figure 9: Decomposition of two details of the test image in Fig. 2. Left column: originals. Second column: cartoon texture for $\sigma=2$. Third column: cartoon and texture for $\sigma=3$. Fourth column: cartoon and texture for $\sigma=4$. 
[20] Y. Gousseau and J.M. Morel. Are natural images of bounded variation? Siam J. Math. Anal., 33:634-648, 2001.

[21] M. Green. Statistics of images, the TV algorithm of Rudin-Osher-Fatemi for image denoising and an improved denoising algorithm. UCLA C.A.M. Report, 02-55, 2002.

[22] A. Gunturk Haddad and Y. Meyer. An improvement of Rudin-Osher-Fatemi model. Applied and Computational Harmonic Analysis, 22(3):319-334, 2007.

[23] T.M. Le and L.A. Vese. Image decomposition using total variation and $\operatorname{div}(B M O)$. SIAM Multiscale Modeling and Simulation, 4(2):390-423, 2005.

[24] S. Levine. An adaptive variatonal model for image decomposition. Lecture Notes in Computer Science, 3757:382-397, 2005.

[25] L.H. Lieu and L.A. Vese. Image restoration and decomposition via bounded total variation and negative Hilbert-Sobolev spaces. Applied Mathematics and Optimization, 58:167-193, 2008.

[26] S. Lintner and F. Malgouyres. Solving a variational image restoration model which involves $L^{\infty}$ constraints. Inverse Problems, 20(3):815-831, 2004.

[27] F. Malgouyres. Mathematical analysis of a model which combines total variation and wavelet for image restoration. Journal of Information Processes, 2(1):1-10, 2002 .

[28] Y. Meyer. Oscillating patterns in image processing and nonlinear evolution equations. American Mathematical Society Providence, RI, 2001.

[29] J.M. Morel and S. Solimini. Variational methods in image segmentation. Progress in Nonlinear Differential Equations and their Applications, 14, 1995.

[30] D. Mumford and J. Shah. Optimal Approximations by Piecewise Smooth Functions and Associated Variational Problems. Center for Intelligent Control Systems, 1988.

[31] D. Mumford and J. Shah. Optimal approximations by piecewise smooth functions and associated variational problems. Comm. Pure Appl. Math., 42(5):577-685, 1989 .

[32] A. Obereder, S. Osher, and O. Scherzer. On the use of dual norms in bounded variation type regularization. in Computational Imaging and Vision: Geometric Properties for Incomplete data, R. Klette, R. Kozera, L. Noakes and J. Weickert (Eds.), pages 373-390, 2006.

[33] S. Osher, M. Burger, D. Goldfarb, J. Xu, and W. Yin. An iterative regularization method for total variation-based image restoration. Multiscale Modeling and Simulation, to appear.

[34] S. Osher, S. Kindermann, and P. Jones. Deblurring and denoising of images by nonlocal functionals. SIAM Multiscale and Simulation, 4(4):1091-1115, 2005. 
[35] S. Osher and O. Scherzer. G-norm properties of bounded variation regularization. Commun. Math. Sci., 2(2):237-254, 2004.

[36] S.J. Osher, A. Solé, and L.A. Vese. Image decomposition and restoration using total variation minimization and the $H^{-1}$ norm. Multiscale Modeling and Simulation, 1(3):349-370, 2003.

[37] L. Rudin, S. Osher, and E. Fatemi. Nonlinear total variation based noise removal algorithms. Physica D, 60(1-4):259-268, 1992.

[38] J.P. Serra. Image analysis and mathematical morphology. Academic Press (London), 1982.

[39] J. Shen. Piecewise $H^{-1}+H^{0}+H^{1}$ images and the Mumford-Shah-Sobolev model for segmented image decomposition. Appl Mathematics Research eXpress, (4):143167, 2005.

[40] J.L. Starck, M. Elad, and D.L. Donoho. Image decomposition: separation of texture from piecewise smooth content. in Proceedings SPIE Annual Meeting, 2003.

[41] D. Strong and T. Chan. Edge-preserving and scale-dependent properties of total variation regularization. Inverse Problems, 19(6):165-187, 2003.

[42] D.M. Strong, J.F. Aujol, and T.F. Chan. Scale recognition, regularization parameter selection, and Meyer's G norm in total variation regularization. Multiscale Modeling and Simulation, a SIAM Journal, 5(1):273-303, 2006.

[43] E. Tadmor, S. Nezzar, and L. Vese. A multiscale image representation using hierarchical $\left(B V, L^{2}\right)$ decompositions. Multiscale Modeling and Simulation, 2:554$579,2004$.

[44] L. Vese. A study in the $B V$ space of a denoising-deblurring variational problem. Applied Mathematics and Optimization, 44(2):131-161, 2001.

[45] L.A. Vese and S. Osher. Image denoising and decomposition with total variation minimization and oscillatory functions. Journal of Mathematical Imaging and Vision, 20(1):7-18, 2004.

[46] L.A. Vese and S.J. Osher. Modeling Textures with Total Variation Minimization and Oscillating Patterns in Image Processing. Journal of Scientific Computing, 19(1):553-572, 2003.

[47] W. Yin, D. Goldfarb, and S. Osher. A comparison of three total variation based texture extraction models. Journal of Visual Communication and Image Representation, 18(3):240-252, 2007. 